\title{
Breakup of Spiral Wave and Order-Disorder Spatial Pattern Transition Induced by Spatially Uniform Cross-Correlated Sine-Wiener Noises in a Regular Network of Hodgkin-Huxley Neurons
}

\author{
Yuangen Yao, ${ }^{1,2}$ Wei Cao, ${ }^{1,3}$ Qiming Pei, ${ }^{4}$ Chengzhang Ma ${ }^{D},{ }^{1}$ and Ming Yi ${ }^{1}$ \\ ${ }^{1}$ Department of Physics, College of Science, Huazhong Agricultural University, Wuhan, China \\ ${ }^{2}$ Institute of Applied Physics, Huazhong Agricultural University, Wuhan, China \\ ${ }^{3}$ Department of Physics and Institute of Biophysics, Central China Normal University, Wuhan, China \\ ${ }^{4}$ School of Physics and Optoelectronic Engineering, Yangtze University, Jingzhou, China \\ Correspondence should be addressed to Chengzhang Ma; ma@mail.hzau.edu.cn
}

Received 28 September 2017; Revised 30 January 2018; Accepted 22 February 2018; Published 22 April 2018

Academic Editor: Daniela Paolotti

Copyright (c) 2018 Yuangen Yao et al. This is an open access article distributed under the Creative Commons Attribution License, which permits unrestricted use, distribution, and reproduction in any medium, provided the original work is properly cited.

\begin{abstract}
Spatially uniform cross-correlated sine-Wiener (CCSW) noises are imposed on each Hodgkin-Huxley (HH) neuron in a twodimensional (2D) regular network. Noise-induced spiral wave destruction and order-disorder spatial pattern transition can be observed by adjusting cross-correlation time or cross-correlation intensity of CCSW noises. The sudden change of the curve of synchronization factor $R$ and the time series of average membrane voltage $F$ can be used to semiquantitatively assess this spatial pattern transition and spiral wave destruction induced by CCSW noises, respectively. In addition, moderate cross-correlation time and strong cross-correlation intensity of CCSW noises are both detrimental to survival of spiral wave. Comparing with non-crosscorrelated sine-Wiener noises, CCSW noises can destroy organized spiral wave with lesser noise amplitude.
\end{abstract}

\section{Introduction}

A hot issue in neurosciences is to investigate synchronized collective behaviors of a group of interacting neurons due to their potential applications in information processing, information transmission, and so on. Synchronized collective behaviors of neurons in a network often manifest spatiotemporal pattern. Spiral wave is one of the most important spatiotemporal patterns and widely observed in various real systems. For example, spiral wave is observed in the cardiac tissue and it relates to a kind of heart disease [1-3]. Therefore, many schemes have been presented to remove and suppress spiral wave in the cardiac tissue [4-6]. Additionally, spiral wave in the neocortex may relate to seizure by extending the duration of evoked activity in pathological conditions $[7,8]$. However, spiral wave in the neocortex can be used as a spatial framework to coordinate cortical oscillations over a group of neurons so that it may contribute to signal communication $[7,8]$. In consideration of importance of spiral wave, it is especially interesting and important to investigate the dynamics of spiral waves in various different systems.

Random fluctuations (also known as noises) are omnipresent in nature [9]. Choosing reasonable model to mimic these random fluctuations is very important for the study of the dynamics of nonlinear systems [10]. Gaussian noise is widely used to model random fluctuations because of its convenience of analysis. However, considering that some real physical quantities, such as reaction rates, must be strictly positive particularly attention must be paid in order to avoid the fact that some undesired and unrealistic modelbased results may occur due to unboundedness of Gaussian noise [11]. Unlike unbounded Gaussian noise, bounded noise takes values in bounded intervals and bounded noise is 
naturally consistent with the boundedness of real physical quantities in real systems [10, 11]. Furthermore, bounded noise is a convenient and natural mathematical tool for modeling either narrow band or broad band random process by appropriately adjusting corresponding parameters [12]. Hence, bounded stochastic processes are increasingly employed in various research fields involving electrical engineering, mechanical and structural engineering, and biological systems [13]. For example, impacts of bounded noise on the formation and instability of spiral waves in regular and small-world networks of Hodgkin-Huxley $(\mathrm{HH})$ neurons were investigated in our previous works [14, 15] and the constructive role of bounded noise in facilitating formation and stability of spiral waves was discovered $[14,15]$.

It is noteworthy that the majority of studies about the impact of bounded noises including our previous works assume that a unique noise or multiple uncorrelated noises are perturbing an otherwise deterministic system. However, there may be correlation between multiple noises if they share a common origin. A few studies on cross-correlated bounded noises have been reported recently. For example, cross-correlated sine-Wiener (CCSW) noises can induce transitions in tumor-immune system interplay [16], genotype selection model [17], and the modified FitzHugh-Nagumo (FHN) neuron model [18]. More interestingly, CCSW noisesinduced transition vanishes if both noises are not correlated [18]. Moreover, CCSW noises-induced coherence resonance (CR) phenomenon is discovered in the FHN neuron. Furthermore, CR is sensitive to the amplitudes and correlation times of CCSW noises [19]. CCSW noises [20] can also greatly enhance weak signal detection and transmission through resonance mechanism like periodic force [21-23], time delay [24, 25], electromagnetic induction [26-31], and sine-Wiener noise [32]. Although some studies on CCSW noises have been achieved, to our best knowledge, the effects of CCSW noises on the spiral wave and pattern dynamics have not been so far reported. To do this, we firstly construct a twodimensional (2D) regular network of $\mathrm{HH}$ neurons subjected to spatially uniform CCSW noises and then investigate spiral wave destruction and spatial pattern transition induced by spatially uniform CCSW noises.

\section{Model and Simulation}

As reported previously, CCSW noises of $\eta_{1}(t)$ and $\eta_{2}(t)$ are described by following formulas $[16,18,33]$ :

$$
\begin{aligned}
& \eta_{1}(t)=\sigma_{1} \sin \left(\sqrt{\frac{2}{\tau_{1}}} \omega_{1}(t)\right), \\
& \eta_{2}(t)=\sigma_{2} \sin \left(\sqrt{\frac{2}{\tau_{2}}} \omega_{2}(t)\right) .
\end{aligned}
$$

Here $\sigma_{1}$ and $\sigma_{2}$ are amplitudes of $\eta_{1}(t)$ and $\eta_{2}(t)$, while $\tau_{1}$ and $\tau_{2}$ are self-correlation times of $\eta_{1}(t)$ and $\eta_{2}(t)$, respectively. Unless other specified, $\sigma_{1}$ and $\sigma_{2}$ are set to 15 throughout this work. $\omega_{1}$ and $\omega_{2}$ are two cross-correlated standard Wiener processes with cross-correlation functions defined as

$$
\left\langle\omega_{1}(t) \omega_{2}\left(t^{\prime}\right)\right\rangle=\left\langle\omega_{1}\left(t^{\prime}\right) \omega_{2}(t)\right\rangle=\lambda \cdot \min \left(t, t^{\prime}\right) .
$$

Here $\lambda$ denotes correlation intensity. The symbol of $\langle\cdot\rangle$ represents an ensemble average and $\min \left(t, t^{\prime}\right)$ means smaller value between $t$ and $t^{\prime}$. For the same correlation times $\left(\tau_{1}=\right.$ $\left.\tau_{2}=\tau\right)$, the cross-correlation function of $\eta_{1}(t)$ and $\eta_{2}(t)$ can be expressed as follows [16]:

$$
\begin{aligned}
& \left\langle\eta_{1}(t) \eta_{2}\left(t^{\prime}\right)\right\rangle=\left\langle\eta_{1}\left(t^{\prime}\right) \eta_{2}(t)\right\rangle \\
& =\lambda^{\prime} \frac{\sigma_{1} \sigma_{2}}{2} \exp \left(-\frac{t-t^{\prime}}{\tau}\right)\left[1-\exp \left(-\frac{4 t^{\prime}}{\tau}\right)\right], \\
& t>t^{\prime}
\end{aligned}
$$

with

$$
\lambda^{\prime}=\exp \left(-\frac{2(1-\lambda) t^{\prime}}{\tau}\right) \frac{1-\exp \left(-(4 \lambda / \tau) t^{\prime}\right)}{1-\exp \left(-(4 / \tau) t^{\prime}\right)}
$$

Here $\lambda^{\prime}\left(0 \leq \lambda^{\prime} \leq 1\right)$ and $\tau$ denote cross-correlation intensity and cross-correlation time of $\eta_{1}(t)$ and $\eta_{2}(t)$, respectively. It is very difficult to directly treat CCSW noises due to the presence of coupling between noises. As reported previously, an existing decoupling procedure is used to equivalently transform (1) into the following formulas [16]:

$$
\begin{aligned}
\xi_{1}(t)= & \sigma_{1} \sin \left(\sqrt{\frac{2}{\tau}} \omega_{\alpha}(t)\right), \\
\xi_{2}(t)= & \sigma_{2} \lambda^{\prime} \sin \left(\sqrt{\frac{2}{\tau}} \omega_{\alpha}(t)\right) \\
& +\sigma_{2} \sqrt{1-\lambda^{\prime 2}} \sin \left(\sqrt{\frac{2}{\tau}} \omega_{\beta}(t)\right) .
\end{aligned}
$$

Herein $\omega_{\alpha}$ and $\omega_{\beta}$ denote two independent standard Wiener processes [34]. They can be numerically simulated by the Euler-Maruyama algorithm in conjunction with the BoxMuller algorithm to generate normally distributed variables $[16,35]$ :

$$
\begin{aligned}
& \omega_{\alpha}(t)=\omega_{\alpha}(t-\Delta t)+\sqrt{-2 \Delta t \ln \chi_{1}} \cos \left(2 \pi \chi_{2}\right), \\
& \omega_{\beta}(t)=\omega_{\beta}(t-\Delta t)+\sqrt{-2 \Delta t \ln \chi_{3}} \cos \left(2 \pi \chi_{4}\right) .
\end{aligned}
$$

Here $\chi_{1}, \chi_{2}, \chi_{3}$, and $\chi_{4}$ are four independent random numbers uniformly distributed between 0 and 1 . 
The mathematical model for a $2 \mathrm{D}$ regular network of $\mathrm{HH}$ neurons subjected to CCSW noises is given by the following coupled differential equations $[36,37]$ :

$$
\begin{aligned}
C_{m} \frac{\mathrm{d} V_{i j}}{\mathrm{~d} t} & \\
= & g_{\mathrm{K}} n_{i j}^{4}\left(V_{\mathrm{K}}-V_{i j}\right)+g_{\mathrm{Na}} m_{i j}^{3} h_{i j}\left(V_{\mathrm{Na}}-V_{i j}\right) \\
& +g_{L}\left(V_{L}-V_{i j}\right) \\
& +D\left(V_{i-1, j}+V_{i, j+1}+V_{i+1, j}+V_{i, j-1}-4 V_{i j}\right)+\xi_{1} \\
& +\xi_{2}, \\
\frac{\mathrm{d} y_{i j}}{\mathrm{~d} t}= & \alpha_{y}\left(V_{i j}\right)\left(1-y_{i j}\right)-\beta_{y}\left(V_{i j}\right) y_{i j}, \quad y=m, h, n, \\
\alpha_{m}= & \frac{0.1\left(V_{i j}+40\right)}{1-\exp \left(-\left(V_{i j}+40\right) / 10\right)}, \\
\beta_{m}= & 4 \exp \left(-\frac{\left(V_{i j}+65\right)}{18}\right), \\
\alpha_{h}= & 0.07 \exp \left(-\frac{\left(V_{i j}+65\right)}{20}\right), \\
\beta_{h}= & \frac{1}{1+\exp \left(-\left(V_{i j}+35\right) / 10\right)}, \\
\alpha_{n}= & \frac{0.01\left(V_{i j}+55\right)}{1-\exp \left(-\left(V_{i j}+55\right) / 10\right)}, \\
& \\
&
\end{aligned}
$$

Here $V_{i j}$ denotes the voltage of the cellular membrane of the neuron at the node $(i, j)$, while $m_{i j}, n_{i j}$, and $h_{i j}$ stand for gate channel parameters of the neuron at the node $(i, j)$. All parameter values used throughout this paper are listed in Table 1.

As reported previously, to measure spatial synchronization, a synchronization factor $R$ is introduced as follows $[14,15,38]$ :

$$
\begin{aligned}
& F=\frac{1}{N^{2}} \sum_{j=1}^{N} \sum_{i=1}^{N} V_{i j}, \\
& R=\frac{\left\langle F^{2}\right\rangle-\langle F\rangle^{2}}{\left(1 / N^{2}\right) \sum_{j=1}^{N} \sum_{i=1}^{N}\left(\left\langle V_{i j}^{2}\right\rangle-\left\langle V_{i j}\right\rangle^{2}\right)} .
\end{aligned}
$$

Here $\langle\cdot\rangle$ represents averaging over time. The value of $R$ indicates the degree of synchronization. Larger $R$ denotes better synchronization. In particular, that $R$ is close to 0 means no synchronization, while that $R$ is close to 1 indicates perfect synchronization. As reported previously, the sudden change
TABLE 1: Employed parameters and their values. Unless otherwise specified, these parameters remain unchanged throughout this paper.

\begin{tabular}{lc}
\hline Parameter names & Parameter values \\
\hline Membrane capacitance $\left(\mu \mathrm{F} / \mathrm{cm}^{2}\right)$ & $C_{m}=1$ \\
Conductance constant for sodium $\left(\mathrm{mS} / \mathrm{cm}^{2}\right)$ & $g_{\mathrm{Na}}=120$ \\
Conductance constant for potassium $\left(\mathrm{mS} / \mathrm{cm}^{2}\right)$ & $g_{\mathrm{K}}=36$ \\
Conductance constant for leakage current & $g_{L}=0.3$ \\
$\left(\mathrm{mS} / \mathrm{cm}^{2}\right)$ & $V_{\mathrm{Na}}=50$ \\
Reversal potential for sodium $(\mathrm{mV})$ & $V_{\mathrm{K}}=-77$ \\
Reversal potential for potassium $(\mathrm{mV})$ & $V_{L}=-54.4$ \\
Reversal potential for leakage current $(\mathrm{mV})$ & $D=0.5$ \\
Coupling intensity between different neurons & $N=100$ \\
Neurons number in the network $(N \times N)$ &
\end{tabular}

of $R$ can be used to indicate synchronization transition [14, 39]. In the presence of CCSW noises, a sufficiently large time window of 500 time units is used in the calculation of synchronization factor $R$.

According to Euler forward difference algorithm, equations of (7) and (8) are solved with the no-flux boundary condition and time step $\Delta t=0.001$ in our numerical simulations. A wedge-shaped initial value method of $V(41: 43,1: 50)=$ -40.2, $V(44: 46,1: 50)=0, V(47: 49,1: 50)=40.0, m(41: 43$, $1: 50)=0.1203, m(44: 46,1: 50)=0.5203, m(47: 49,1: 50)=$ $0.98203, h(41: 43,1: 50)=0.9, h(44: 46,1: 50)=0.7, h(47: 49$, $1: 50)=0.5, n(41: 43,1: 50)=0.9, n(44: 46,1: 50)=0.7$, and $n(47: 49,1: 50)=0.5$ is used to induce a spiral seed, while the initial values for other neurons are selected with $V(i, j)=$ -61.19389, $m(i, j)=0.08203, h(i, j)=0.46012$, and $n(i, j)=$ 0.37726 . In the absence of CCSW noises, a sufficiently large time window of 500 time units is used in order to ensure obtaining a perfect spiral wave. CCSW noises are introduced into deterministic system at a given time instant of $t=500$ time units, and then CCSW noises are persistently imposed on each $\mathrm{HH}$ neuron in the system.

\section{Main Results}

This study focuses primarily on spiral wave destruction and spatial pattern transition induced by spatially uniform CCSW noises. Therefore, the effect of cross-correlation time $\tau$ of CCSW noises is firstly investigated. As shown in Figure 1, for small cross-correlation time $\tau$ (such as $\log _{10}(\tau)=-0.5,0$, and 0.5 ), spiral wave can survive under the disturbance of CCSW noises (Figures 1(a) and 1(b)). However, with the increase of cross-correlation time $\tau$, spiral wave starts to be destroyed (Figure 1(c)). If cross-correlation time is large enough, CCSW noises can completely destroy spiral wave and result in the occurrence of irregular pattern (Figures 1(d)-1(e)). Interestingly, if cross-correlation time $\tau$ of CCSW noises is further increased, spiral wave actually keeps its alive (Figures 1(h)1(i)). Therefore, moderate cross-correlation time of CCSW noises is detrimental to survival of spiral wave. Moreover, CCSW noises-induced order-disorder spatial pattern transitions from spiral wave to irregular pattern can be obtained by 


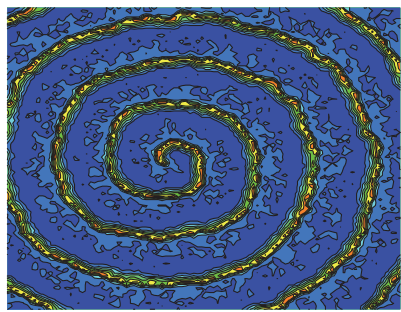

(a)

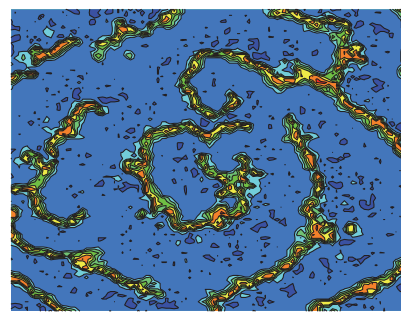

(d)
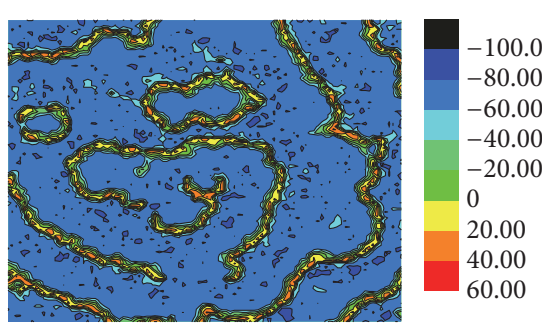

(g)
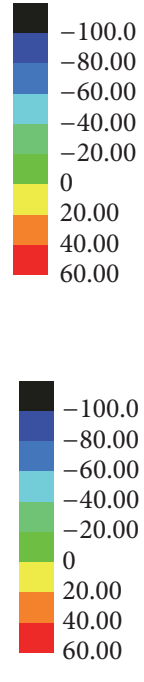

0.00

(6)

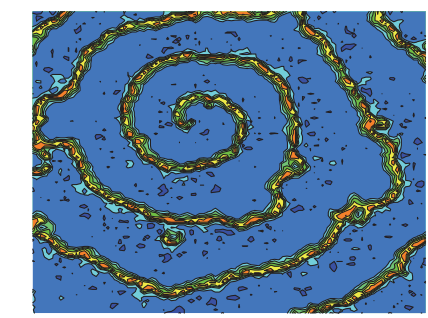

(h)

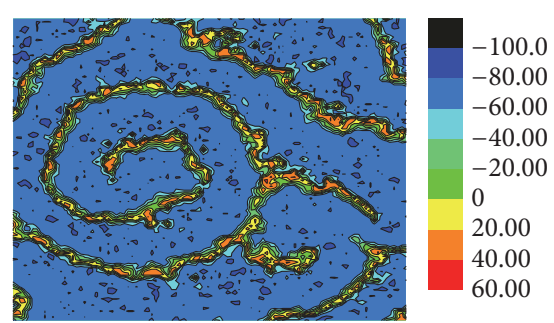

(c)

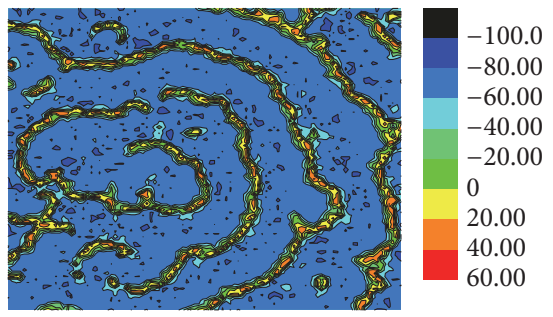

(f)

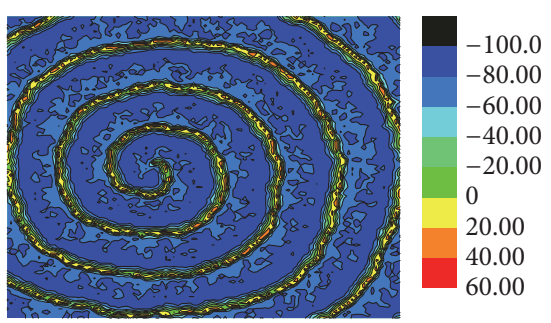

(i)

FIGURE 1: Noises-induced pattern transition obtained by adjusting cross-correlation time of CCSW noises. $\lambda^{\prime}=0.5 \cdot \log _{10}(\tau)=-0.5(\mathrm{a}), 0$ (b), 0.5 (c), 1 (d), 1.5 (e), 2 (f), 2.5 (g), 3 (h), and 3.5 (i).

adjusting cross-correlation time $\tau$ of CCSW noises. Besides qualitative observation from snapshots of spatial pattern, this transition process can be semiquantitatively assessed by the sudden change of the curve of $R$. As displayed in Figure 2, that $R$ sharply increases at first and then decreases rapidly as crosscorrelation time $\tau$ increases results in an obvious peak in the curve of $R-\log _{10}(\tau)$, which suggests spatial pattern transition from spiral wave to irregular pattern (Figure 2).

It is also interesting and important to observe dynamic process of CCSW noises-induced spiral wave destruction. The snapshots in Figure 3 show the breakup of spiral wave under the disturbance of CCSW noises. Without CCSW noises (Figure 3(a)), a perfect spiral wave can be observed in the network (Figure 3(a)). In the presence of CCSW noises, spiral wave starts to be destroyed by CCSW noises (Figure 3). Finally, spiral wave is almost completely destroyed and irregular spatial pattern appears in the network under the persistent disturbance of CCSW noises (Figure 3). Similarly, in addition to qualitative observation from snapshots of spatial pattern, spiral wave destruction can be identified by analyzing the time series of average membrane voltage $F$. Before CCSW noises are applied to deterministic FHN model, stable spiral wave (indicated by stable periodical oscillation) can be observed in the network over a period of about 200 time units from running (Figure 4). After CCSW noises are imposed on each neuron, spatial pattern

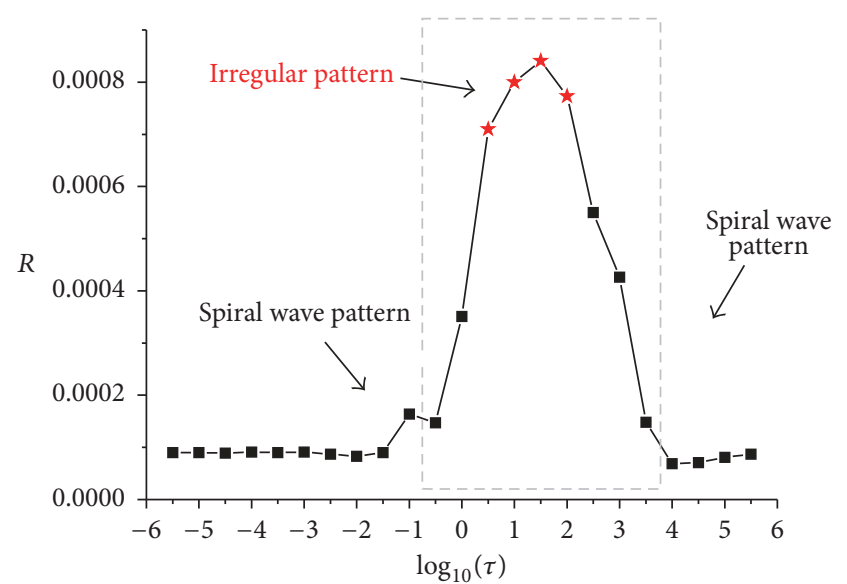

FIgURE 2: The synchronization factor $\mathrm{R}$ as a function of crosscorrelation time $\log _{10}(\tau)$ of CCSW noises. $\lambda^{\prime}=0.5$. The regular spiral wave pattern is indicated by the square symbol, while the irregular pattern is denoted by star symbol. The square and star symbols in the rectangular area surrounded by dashed gray line correspond to the spatial patterns in Figure 1.

is under disturbance of CCSW noises and spiral wave may be destroyed to some extent. For small cross-correlation time of CCSW noises, time series of $F$ presents quasi-periodical 




(a)

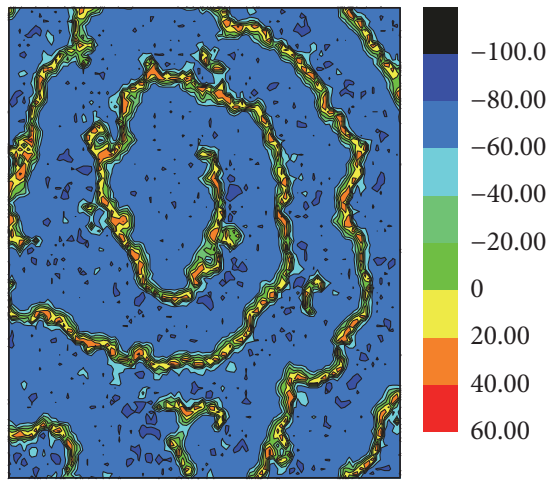

(d)



(b)

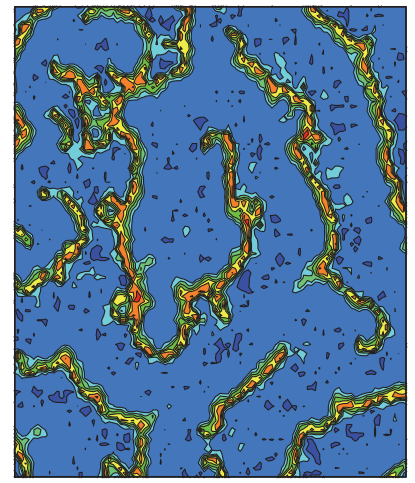

(e)
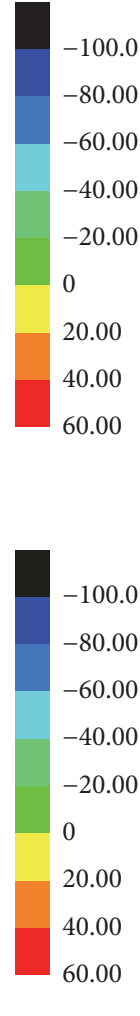

60.00



(c)

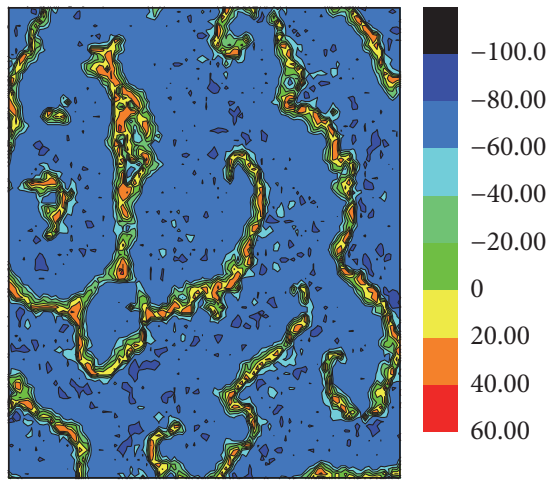

(f)

FIGURE 3: Evolution of spatial pattern within a time window of 500 time units after CCSW noises are imposed on each neuron. $\lambda^{\prime}=0.5$, and $\log _{10}(\tau)=1.5 . t=500(\mathrm{a}), 600$ (b), 700 (c), 800 (d), 900 (e), and 1000 (f) time units. CCSW noises are persistently imposed on each neuron after $t=500$ time units.

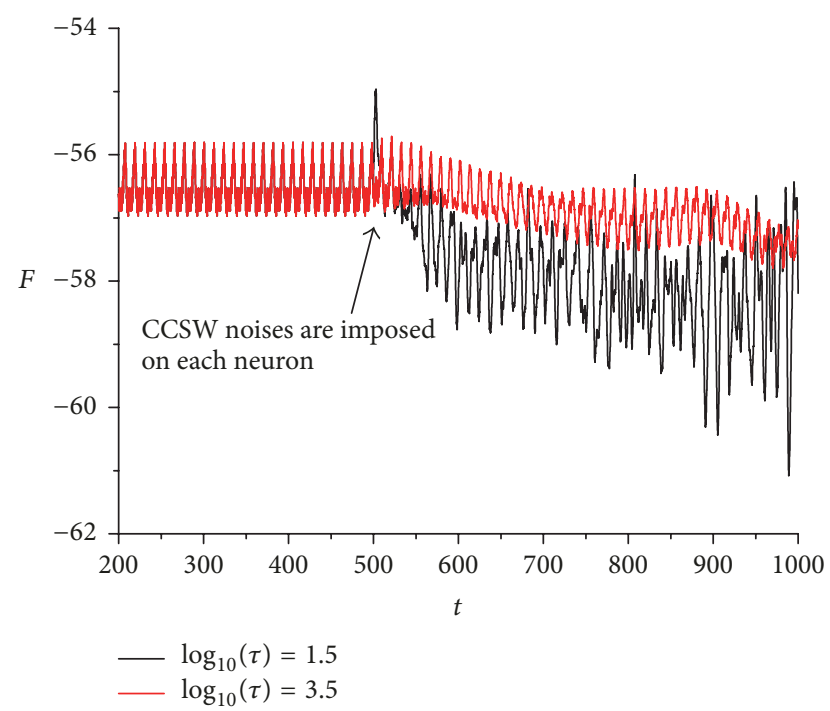

FIGURE 4: Sampled time series of order parameter $\mathrm{F}$ for different cross-correlation time $\log _{10}(\tau)$ of CCSW noises. $\lambda^{\prime}=0.5$.

oscillation, which indicates that spiral wave can survive (Figure 4). For large enough cross-correlation of CCSW noises, time series of $F$ become arbitrary, which denotes CCSW noises-induced spiral wave destruction (Figure 4).
Besides cross-correlation time, cross-correlation intensity $\lambda^{\prime}$ of CCSW noises is a key factor to significantly influence spatial pattern. Therefore, it is also interesting and important to investigate the effect of cross-correlation intensity of CCSW noises. For weak cross-correlation intensity $\lambda^{\prime}$ of CCSW noises, spiral wave can keep it alive under noise disturbance (Figures 5(a) and 5(b)). With the increase of crosscorrelation intensity $\lambda^{\prime}$ of CCSW noises, CCSW noises may destroy regular spiral wave and cause formation of irregular pattern (Figure 5(c)). Interestingly, further increasing crosscorrelation intensity $\lambda^{\prime}$ of CCSW noises does not always destroy spiral wave. For some certain moderate $\lambda^{\prime}$, spiral wave can survive under the noise disturbance (Figures 5(d) and $5(\mathrm{e})$ ). Of course, spiral wave can be completely destroyed for a strong enough cross-correlation intensity $\lambda^{\prime}$ (Figures $5(f)-5(i))$. In other words, strong cross-correlation intensity of CCSW noises is detrimental to survival of spiral wave. In brief, the results illustrated in Figure 5 show noise-induced spiral wave destruction and pattern transition from regular spiral wave to irregular pattern due to the increase of crosscorrelation intensity $\lambda^{\prime}$ of CCSW noises. As described above, synchronization factor $R$ can be used to semiquantificationally assess noise-induced spatial pattern transition. As shown in Figure 6, small $R$ denotes the survival of spiral wave in the condition of weak cross-correlation intensity $\lambda^{\prime}$, while large 


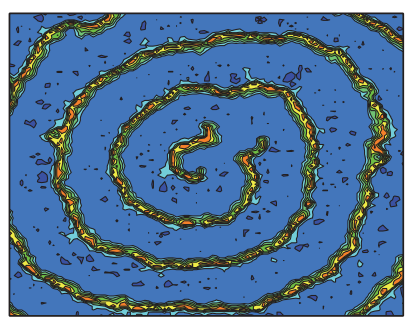

(a)
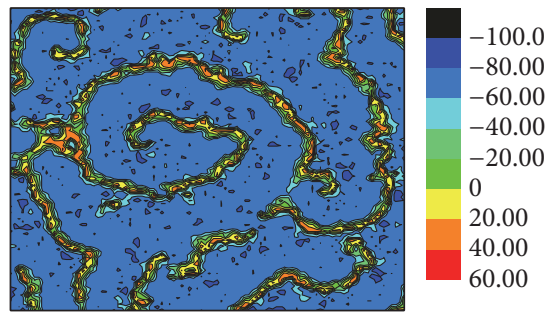

(d)


(g)

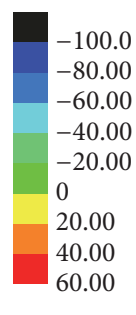

0.00
0.00



(b)

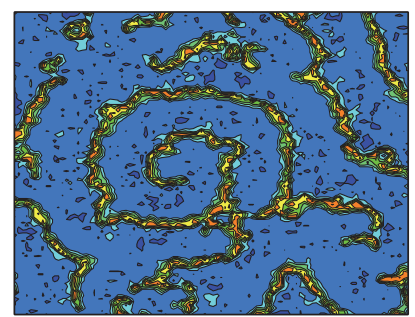

(e)

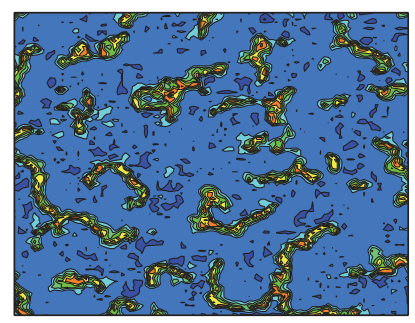

(h)


60.00

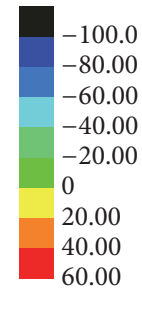

60.00
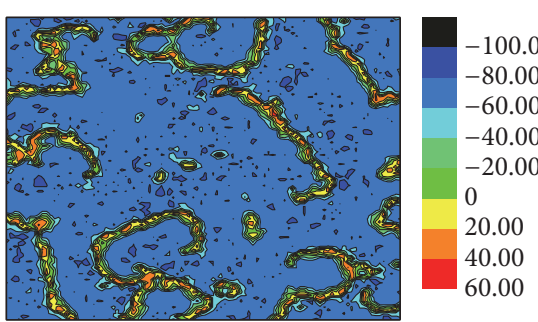

(c)

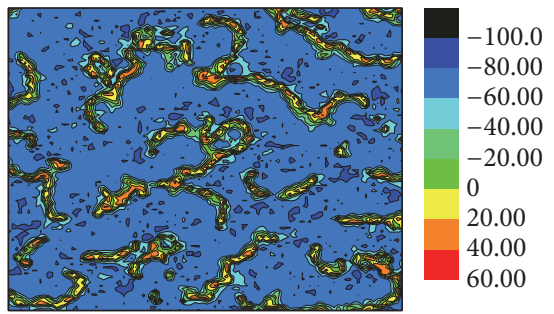

(f)



(i)

FIGURE 5: Noises-induced pattern transition obtained by adjusting cross-correlation intensity $\lambda^{\prime}$ of CCSW noises. $\log _{10}(\tau)=1.5$.

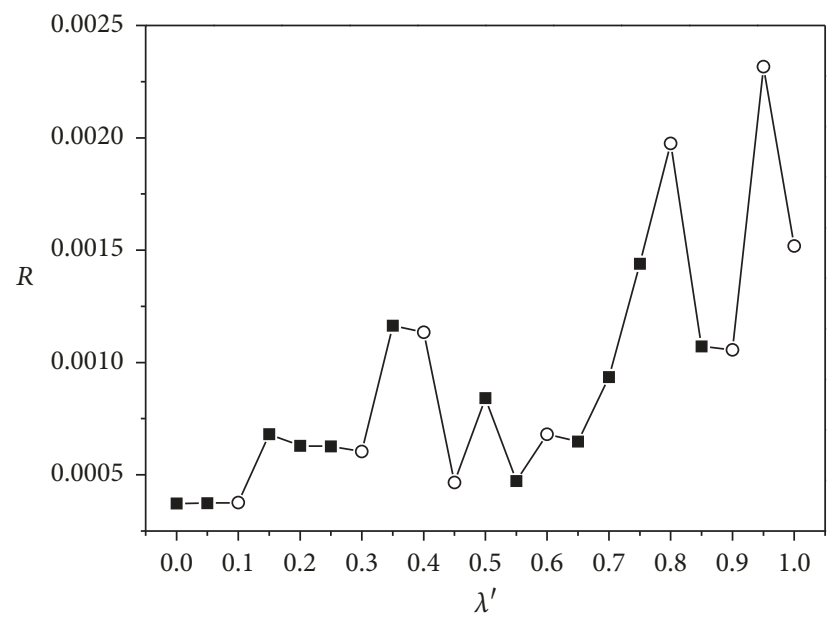

FIgURE 6: The synchronization factor $\mathrm{R}$ as a function of crosscorrelation intensity $\lambda^{\prime}$ of CCSW noises. $\log _{10}(\tau)=1.5$. The open circles correspond to the spatial patterns in Figure 5.

$R$ indicates the breakup of spiral wave and the formation of irregular pattern (Figure 6).

Similarly, we also consider evolution process of spiral wave under the disturbance of CCSW noises. Spiral wave can be clearly observed in the initial transient process after
CCSW noises are applied to network (Figures 7(a) and 7(b)). As time goes on, spiral wave pattern is gradually destroyed and irregular pattern is gradually generated (Figures $7(\mathrm{c})$ and $7(\mathrm{~d})$ ). Finally, spiral wave disappears (Figures $7(\mathrm{e})$ and $7(\mathrm{f})$ ). The corresponding plot of $F$ is drawn in Figure 8. Time series of $F$ presents stable and regular oscillation if without CCSW noises in system, while time series of $F$ starts to become arbitrary if with CCSW noises in system, which suggests spiral wave is being destroyed by CCSW noises (Figure 8).

To get a global view, the contour plot of $R$ in the $\log _{10}(\tau)$ $\lambda^{\prime}$ plane is depicted in Figure 9. As indicated by the blue area with smaller $R$ value, too short or long cross-correlation time $\tau$ of CCSW noises is not fatal for spiral wave. On the whole, for moderate cross-correlation time $\tau, R$ increases gradually along with the increase of cross-correlation intensity $\lambda^{\prime}$ of CCSW noises (Figure 9). However, when $\lambda^{\prime}$ or $\log _{10}(\tau)$ is fixed, the change of $R$ is not always monotonous, which indicates the occurrence of CCSW noises-induced spatial transition (Figure 9). As shown in Figure 9, strong crosscorrelation intensity between noises results in large $R$, which suggests strong cross-correlation intensity is detrimental to the survival of spiral wave. This conclusion can be further confirmed by comparing CCSW noises with non-crosscorrelated sine-Wiener (UNCCSW) noises. Dependence of $R$ on increasing noise amplitudes $\sigma_{1}$ and $\sigma_{2}$ is plotted in Figure 10 for CCSW and UNCCSW noises. $\sigma_{2}$ is assumed 

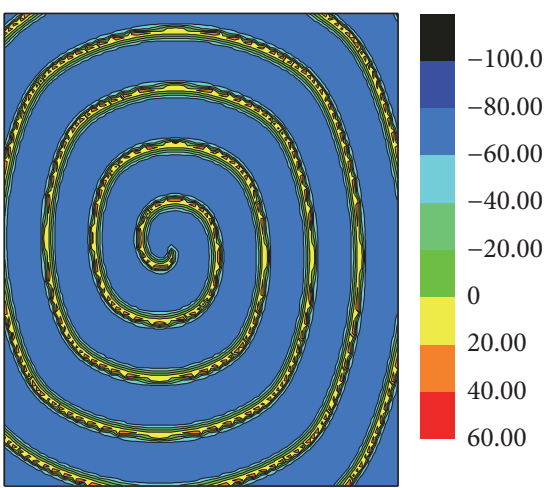

(a)

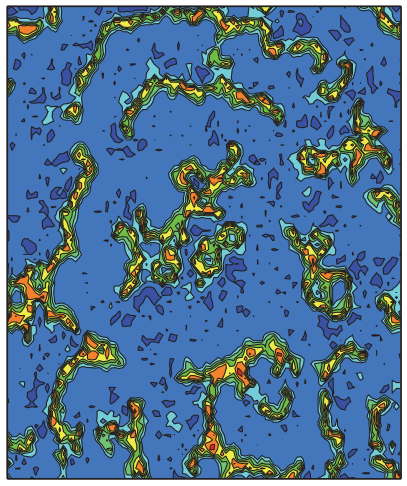

(d)

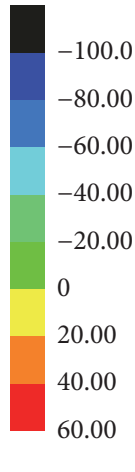

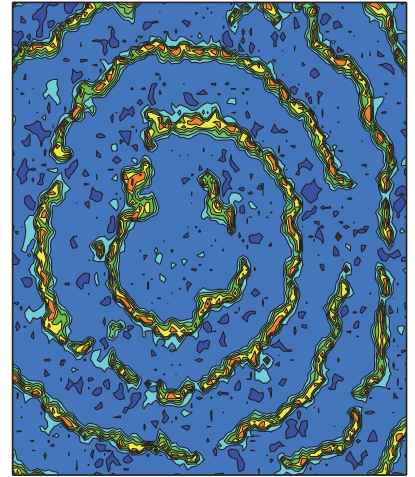

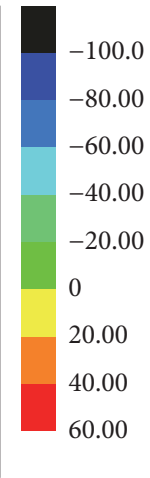

(b)
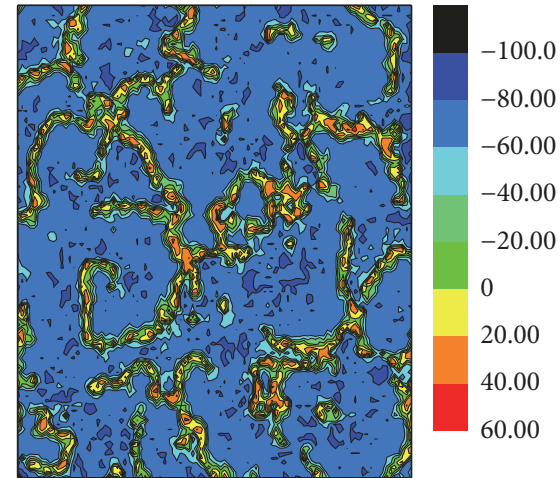

(e)

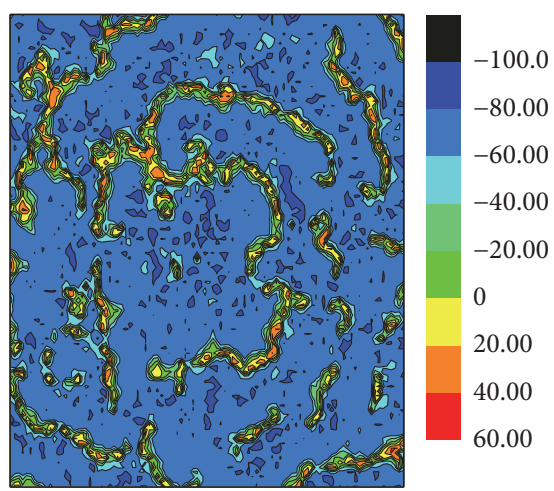

(c)

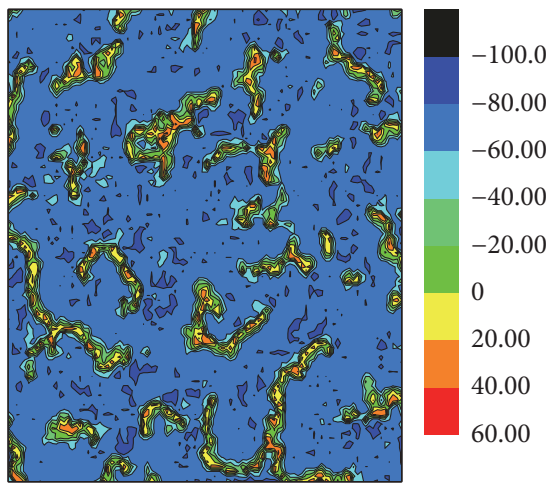

(f)

FIGURE 7: Evolution of spatial pattern within a time window of 500 time units after CCSW noises are imposed on each neuron. $\lambda^{\prime}=0.95$, and $\log _{10}(\tau)=1.5 . t=500(\mathrm{a}), 600(\mathrm{~b}), 700(\mathrm{c}), 800(\mathrm{~d}), 900(\mathrm{e})$, and 1000 (f) time units. CCSW noises are persistently imposed on each neuron after $t=500$ time units.

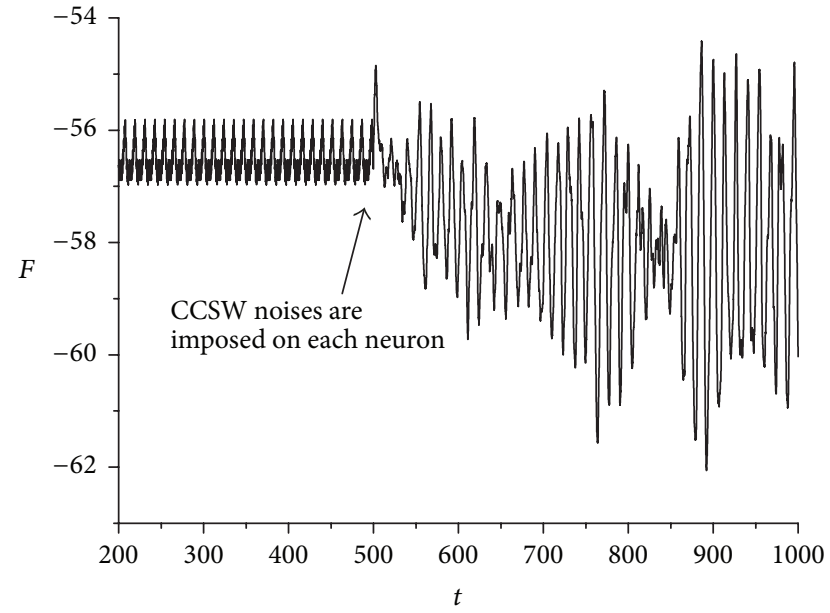

FIGURE 8: Sampled time series of order parameter F. $\lambda^{\prime}=0.95$, and $\log _{10}(\tau)=1.5$.

to be equal to $\sigma_{1}$ in Figure 10 for the sake of the convenient comparison. The value of $R$ for CCSW noises is larger than that for UNCCSW noises (Figure 10). The dividing line between regular spiral wave and irregular pattern is near 0.00085 . For CCSW noises, the threshold of noise amplitude $\sigma_{1}$ for destroying the organized spiral wave is distinctly

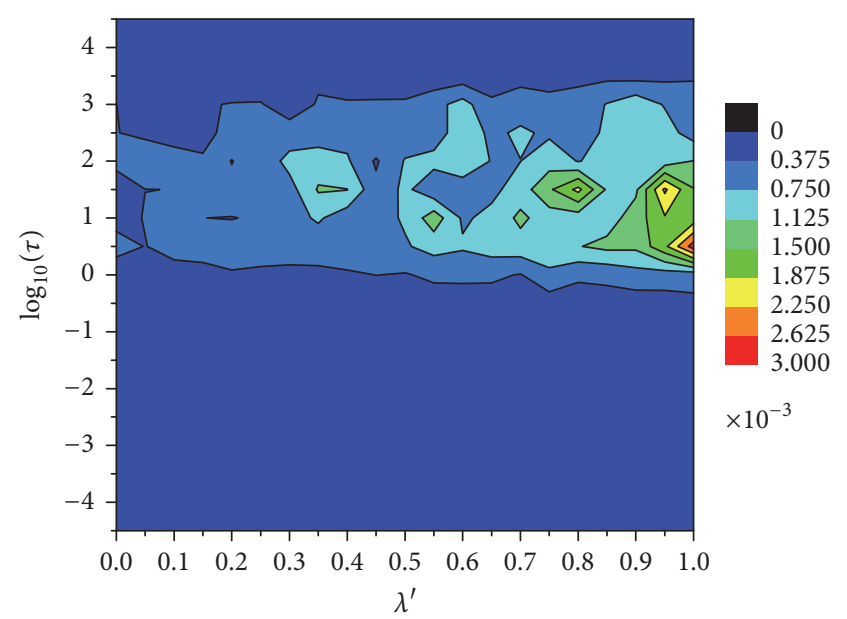

FIGURE 9: Contour plot of synchronization factor $\mathrm{R}$ in the $\log _{10}(\tau)-$ $\lambda^{\prime}$ plane.

less than that for UNCCSW noises (Figure 10). That is to say, cross-correlation between CCSW noises may induce instability of spiral wave and formation of irregular pattern.

In what follows, we investigate how noise amplitudes of CCSW noises affect spatial pattern. Synchronization factor 


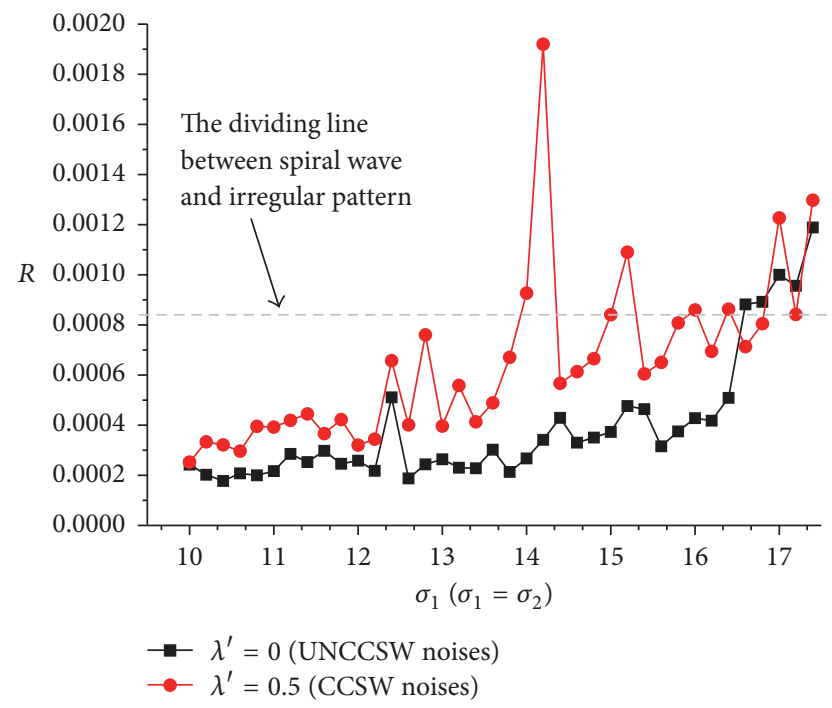

Figure 10: Dependency of synchronization factor $\mathrm{R}$ on noise amplitudes $\sigma_{1}$ and $\sigma_{2}$ for CCSW noises and non-cross-correlated sine-Wiener (UNCCSW) noises. $\sigma_{2}$ is assumed to be equal to $\sigma_{1}$ for the sake of the convenient comparison. $\log _{10}(\tau)=1.5$.

$R$ is calculated for different noise amplitude $\sigma_{2}$ when $\sigma_{1}$ is set to 15 . When noise amplitude $\sigma_{2}$ is small, the value of $R$ is smaller, which denotes that spiral wave can survive under the disturbance of CCSW noises (Figure 11(a)). When noise amplitude $\sigma_{2}$ is large enough, the value of $R$ is larger, which indicates that spiral wave can be destroyed by CCSW noises (Figure 11(a)). However, the change of $R$ with increasing noise amplitude $\sigma_{2}$ is nonmonotonic (Figure 11(a)). Similarly, a contour plot of $R$ in the $\sigma_{2}-\sigma_{1}$ plane is drawn to provide a global view. The blue area with smaller $R$ appears in the lower left corner of the contour plot of $R$, which indicates the survival of spiral wave. The area with larger $R$ in the upper right corner of the contour plot of $R$ indicates disappearance of spiral wave and appearance of irregular pattern due to too large noise disturbance (Figure 11(b)). As a whole, $R$ increases gradually along with the increase of noise amplitudes $\sigma_{1}$ and $\sigma_{2}$ of CCSW noises (Figure 11(b)), but the increase of $R$ is not monotonous with the increase of $\sigma_{1}$ or $\sigma_{2}$ (Figure 11(b)). Although the two cross-correlated bounded noises are in the symmetric form as shown in formulas of (1), the contour plot is not strictly symmetrical along the diagonal, which can be explained by the asymmetry of amplitudes $\sigma_{1}$ and $\sigma_{2}$ in the formulas of (5). The asymmetry is introduced when we indirectly and equivalently deal with CCSW noises by an existing decoupling method.

\section{Discussions and Conclusions}

In summary, spatially uniform CCSW noises are persistently imposed on each $\mathrm{HH}$ neuron in 2D regular network after spiral wave is developed in the network by a wedge-shaped initial value scheme. Then the effects of CCSW noises on spiral wave destruction and spatial pattern transition are investigated. The obtained main results are concluded below. (1) Spatial pattern transitions from spiral wave to irregular pattern can be observed by adjusting cross-correlation time $\tau$ or cross-correlation intensity $\lambda^{\prime}$ of CCSW noises and this CCSW noises-induced spatial pattern transition can be semiquantitatively assessed by the sudden change of the curve of $R$. (2) The regular and irregular oscillation of time series of average membrane voltage $F$ correspond, respectively, to regular spiral wave and irregular pattern. Therefore, CCSW noises-induced spiral wave destruction can be indicated by time series of statistical parameter $F$. (3) For small or large cross-correlation time of CCSW noises, spiral wave may survive. For moderate cross-correlation time of CCSW noises, spiral wave may be destroyed. (4) Strong crosscorrelation intensity between CCSW noises is detrimental to the survival of spiral wave. In particular, comparing with non-cross-correlated sine-Wiener noises, CCSW noises can destroy spiral wave with lesser noise amplitude.

The network model in this work is a most widely adopted one. However, some limitations should be pointed out. The first one is that the coupling between neurons is bidirectional. In fact, neuronal networks are mainly coupled by chemical synapses, which is one directional coupling from presynaptic to postsynaptic neuron. The second one is that temporal delay is negligible in this study. In reality, time delay is inherent in realistic neuronal network due to finite propagation velocities of information. There is a time delay of $\sim 1 \mathrm{~ms}$ between the presynaptic action potential and postsynaptic depolarization. However, in cardiac tissue the coupling is mainly gap junctions, the coupling is bidirectional, and there is less time delay. The third one is that topology structure of network is not considered in our model. Therefore, the network model we used here is a generalized and idealized mathematical model for neuronal network. We believe that idealization procedure of model does not affect the main results we report here. In the future work we will extend our current research to a more complex network model that includes more details such as time delay and complex network topology.

We only considered the temporal correlation between bounded noises. CCSW noises are assumed to be spatially uniform in 2D regular network, which implies a strong unrealistic assumption: the correlation length of the noises is infinite. It is more realistic and reasonable to introduce spatiotemporal Gaussian or bounded noises when investigating dynamics of spatially extended systems subjected to random disturbance. There have been several landmark studies about spatiotemporal noises [11, 40-46]. In particular, spatiotemporal sine-Wiener bounded noise was proposed and its effect on Ginzburg-Landau model was investigated [43]. The emergence of noise-induced reentrant transitions was revealed through numerical simulations [43]. Furthermore, there have been some representative works that apply spatiotemporal bounded noises to a spatial biological problem. For instance, cell polarization may be destroyed when external spatiotemporal bounded noise is introduced into model [44].

In this work, we used the sudden change of the curve of $R$ to semiquantitatively assess pattern transition. More detailed quantitative comparison could be obtained by using the bivariate Moran index $[42,47]$. Note that since $R$ and $F$ are two statistics, more information could be inferred by 


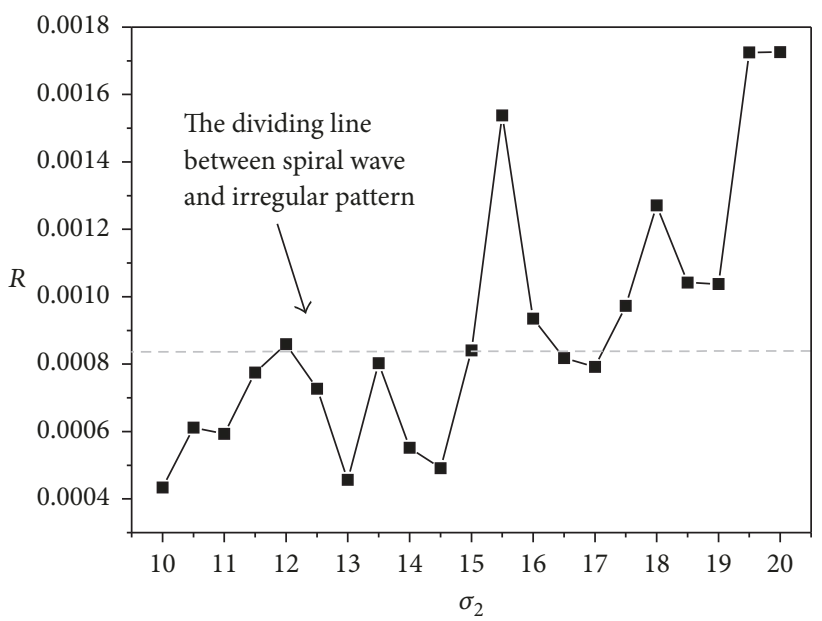

(a)

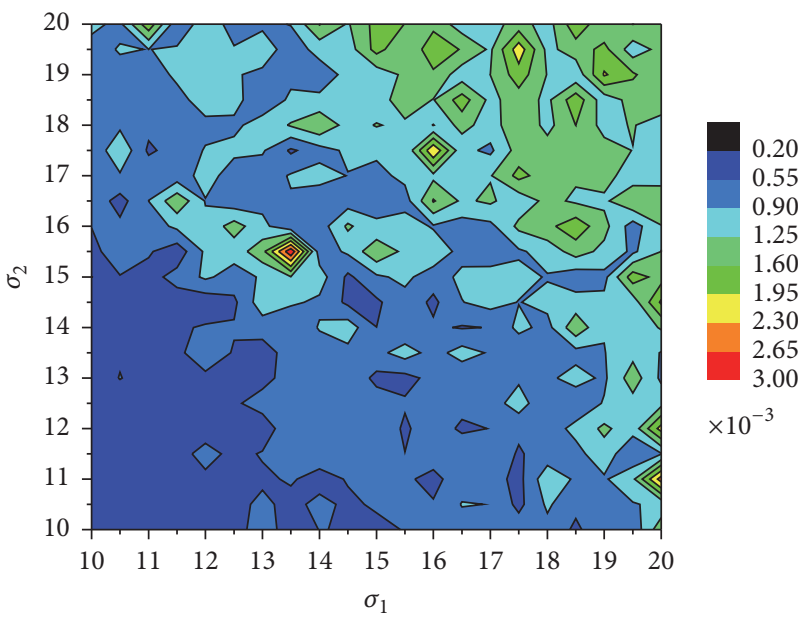

(b)

FIGURE 11: Dependency of synchronization factor R on noise amplitudes of CCSW noises. (a) The synchronization factor $R$ as a function of noise amplitude $\sigma_{2} . \sigma_{1}=15, \lambda^{\prime}=0.5$, and $\log _{10}(\tau)=1.5$; (b) Contour plot of synchronization factor $R$ in the $\sigma_{2}-\sigma_{1}$ plane. $\lambda^{\prime}=0.5$, and $\log _{10}(\tau)=1.5$

plotting, at a given time instant and for various values of the parameters of interest, the histograms of the whole distribution of the fields as in $[42,43]$. The procedure allows better understanding of how the parameters of a spatiotemporal noise impact on the whole patterns of the output of the perturbed system. In future studies we will extend our current research from CCSW noises to spatiotemporal bounded noises and adopt more detailed quantitative methods for better presentation of research findings.

\section{Conflicts of Interest}

The authors declare that they have no conflicts of interest.

\section{Acknowledgments}

This work was supported by the National Natural Science Foundation of China (Grant nos. 31601071, 11605014, and $11675060)$ and the Huazhong Agricultural University Scientific \& Technological Self-Innovation Foundation (Grant no. 2015RC021).

\section{References}

[1] R. A. Gray, J. Jalife, A. V. Panfilov et al., "Mechanisms of cardiac fibrillation," Science, vol. 270, no. 5239, pp. 1222-1225, 1995.

[2] F. X. Wltkowskl, L. J. Leon, P. A. Penkoske et al., "Spatiotemporal evolution of ventricular fibrillation," Nature, vol. 392, no. 6671, pp. 78-82, 1998.

[3] J. Jalife, "Ventricular fibrillation: Mechanisms of iniation maintenance," Annual Review of Physiology, vol. 62, pp. 25-50, 2000.

[4] S. Alonso, F. Sagués, and A. S. Mikhailov, "Taming winfree turbulence of scroll waves in excitable media," Science, vol. 299, no. 5613, pp. 1722-1725, 2003.

[5] H. Zhang, Z. Cao, N.-J. Wu, H.-P. Ying, and G. Hu, "Suppress winfree turbulence by local forcing excitable systems," Physical Review Letters, vol. 94, no. 18, Article ID 188301, 2005.
[6] N.-J. Wu, H. Zhang, H.-P. Ying, Z. Cao, and G. Hu, "Suppression of Winfree turbulence under weak spatiotemporal perturbation," Physical Review E: Statistical, Nonlinear, and Soft Matter Physics, vol. 73, no. 6, Article ID 060901, 2006.

[7] X. Huang, W. C. Troy, Q. Yang et al., "Spiral waves in disinhibited mammalian neocortex," The Journal of Neuroscience, vol. 24, no. 44, pp. 9897-9902, 2004.

[8] X. Huang, W. Xu, J. Liang, K. Takagaki, X. Gao, and J.-Y. Wu, "Spiral Wave Dynamics in Neocortex," Neuron, vol. 68, no. 5, pp. 978-990, 2010.

[9] A. A. Faisal, L. P. J. Selen, and D. M. Wolpert, "Noise in the nervous system," Nature Reviews Neuroscience, vol. 9, no. 4, pp. 292-303, 2008

[10] G. Q. Cai and C. Wu, "Modeling of bounded stochastic processes," Probabilistic Engineering Mechanics, vol. 19, no. 3, pp. 197-203, 2004.

[11] S. de Franciscis, G. Caravagna, and A. d'Onofrio, "Bounded noises as a natural tool to model extrinsic fluctuations in biomolecular networks," Natural Computing, vol. 13, no. 3, pp. 297-307, 2014.

[12] X. L. Yang, Y. B. Jia, and L. Zhang, "Impact of bounded noise and shortcuts on the spatiotemporal dynamics of neuronal networks," Physica A: Statistical Mechanics and its Applications, vol. 393, pp. 617-623, 2014.

[13] A. d'Onofrio, Ed., Bounded Noises in Physics, Biology, and Engineering, Modeling and Simulation in Science, Engineering and Technology, Birkhäuser, Boston, MA, USA, 2013.

[14] Y. Yao, H. Deng, M. Yi, and J. Ma, "Impact of bounded noise on the formation and instability of spiral wave in a 2D Lattice of neurons," Scientific Reports, vol. 7, Article ID 43151, 2017.

[15] Y. Yao, H. Deng, C. Ma, M. Yi, and J. Ma, "Impact of bounded noise and rewiring on the formation and instability of spiral waves in a small-world network of hodgkin-huxley neurons," PLoS ONE, vol. 12, no. 1, Article ID e0171273, 2017.

[16] W. Guo, L.-C. Du, and D.-C. Mei, "Transitions induced by time delays and cross-correlated sine-Wiener noises in a tumorimmune system interplay," Physica A: Statistical Mechanics and its Applications, vol. 391, no. 4, pp. 1270-1280, 2012. 
[17] P. Liu and L. J. Ning, "Transitions induced by cross-correlated bounded noises and time delay in a genotype selection model," Physica A: Statistical Mechanics and its Applications, vol. 441, Article ID 16352, pp. 32-39, 2016.

[18] D. Tatchim Bemmo, M. Siewe Siewe, and C. Tchawoua, "Combined effects of correlated bounded noises and weak periodic signal input in the modified FitzHugh-Nagumo neural model," Communications in Nonlinear Science and Numerical Simulation, vol. 18, no. 5, pp. 1275-1287, 2013.

[19] Y. Yao, M. Yi, and D. Hou, "Coherence resonance induced by cross-correlated sine-Wiener noises in the FitzHugh-Nagumo neurons," International Journal of Modern Physics B, vol. 31, no. 28, Article ID 1750204, 2017.

[20] Y. Yao, C. Ma, C. Wang, M. Yi, and R. Gui, "Detection of sub-threshold periodic signal by multiplicative and additive cross-correlated sine-Wiener noises in the FitzHugh-Nagumo neuron," Physica A: Statistical Mechanics and its Applications, vol. 492, pp. 1247-1256, 2018.

[21] C. Yao and M. Zhan, "Signal transmission by vibrational resonance in one-way coupled bistable systems," Physical Review E: Statistical, Nonlinear, and Soft Matter Physics, vol. 81, no. 6, Article ID 061129, 2010.

[22] C. Yao, Z. He, J. Luo, and J. Shuai, "Resonance induced by a spatially periodic force in the reaction-diffusion system," Physical Review E: Statistical, Nonlinear, and Soft Matter Physics, vol. 91, no. 5, Article ID 052901, 2015.

[23] L. Yang, W. Liu, M. Yi et al., "Vibrational resonance induced by transition of phase-locking modes in excitable systems," Physical Review E: Statistical, Nonlinear, and Soft Matter Physics, vol. 86, no. 1, Article ID 016209, 2012.

[24] C. Yao, J. Ma, C. Li, and Z. He, "The effect of process delay on dynamical behaviors in a self-feedback nonlinear oscillator," Communications in Nonlinear Science and Numerical Simulation, vol. 39, pp. 99-107, 2016.

[25] L.-F. Wang, K. Qiu, and Y. Jia, "Effects of time delays in a mathematical bone model," Chinese Physics B, vol. 26, no. 3, Article ID 030503, 2017.

[26] J. Ma, L. Mi, P. Zhou, Y. Xu, and T. Hayat, "Phase synchronization between two neurons induced by coupling of electromagnetic field," Applied Mathematics and Computation, vol. 307, pp. 321-328, 2017.

[27] F. Wu, C. Wang, W. Jin, and J. Ma, "Dynamical responses in a new neuron model subjected to electromagnetic induction and phase noise," Physica A: Statistical Mechanics and its Applications, vol. 469, pp. 81-88, 2017.

[28] J. Ma, F. Wu, T. Hayat, P. Zhou, and J. Tang, "Electromagnetic induction and radiation-induced abnormality of wave propagation in excitable media," Physica A: Statistical Mechanics and its Applications, vol. 486, pp. 508-516, 2017.

[29] M. Ge, Y. Jia, Y. Xu, and L. Yang, "Mode transition in electrical activities of neuron driven by high and low frequency stimulus in the presence of electromagnetic induction and radiation," Nonlinear Dynamics, vol. 91, no. 1, pp. 515-523, 2018.

[30] L. Lu, Y. Jia, W. Liu, and L. Yang, "Mixed stimulus-induced mode selection in neural activity driven by high and low frequency current under electromagnetic radiation," Complexity, vol. 2017, Article ID 7628537, 2017.

[31] Y. Xu, Y. Jia, M. Ge, L. Lu, L. Yang, and X. Zhan, "Effects of ion channel blocks on electrical activity of stochastic Hodgkin-Huxley neural network under electromagnetic induction," Neurocomputing, vol. 283, pp. 196-204, 2018.
[32] Y. Yao and J. Ma, "Weak periodic signal detection by sineWiener-noise-induced resonance in the FitzHugh-Nagumo neuron," Cognitive Neurodynamics, 2018.

[33] R. V. Bobryk and A. Chrzeszczyk, "Transitions induced by bounded noise," Physica A: Statistical Mechanics and its Applications, vol. 358, no. 2-4, pp. 263-272, 2005.

[34] C. W. Gardiner, Handbook of stochastic processes, SpringerVerlag, New York, NY, USA, 1985.

[35] G. E. Box and M. E. Muller, "A note on the generation of random normal deviates," The Annals of Mathematical Statistics, vol. 29, no. 2, pp. 610-611, 1958.

[36] A. L. Hodgkin and A. F. Huxley, "A quantitative description of membrane current and its application to conduction and excitation in nerve., The Journal of Physiology, vol. 117, no. 4, pp. 500-544, 1952.

[37] D. Sterratt, B. Graham, A. Gillies, and D. Willshaw, "Principles of computational modelling in neuroscience," Principles of Computational Modelling in Neuroscience, pp. 1-390, 2011.

[38] J. Ma, H. Qin, X. Song, and R. Chu, "Pattern selection in neuronal network driven by electric autapses with diversity in time delays," International Journal of Modern Physics B, vol. 29, no. 1, Article ID 1450239, 2015.

[39] J. Ma, Y. Wu, H. Ying, and Y. Jia, "Channel noise-induced phase transition of spiral wave in networks of Hodgkin-Huxley neurons," Chinese Science Bulletin, vol. 56, no. 2, pp. 151-157, 2011.

[40] F. Sagués, J. M. Sancho, and J. García-Ojalvo, "Spatiotemporal order out of noise," Reviews of Modern Physics, vol. 79, no. 3, pp. 829-882, 2007.

[41] C. R. Doering, "A stochastic partial differential equation with multiplicative noise," Physics Letters A, vol. 122, no. 3-4, pp. 133139, 1987.

[42] S. De Franciscis and A. D’Onofrio, "Spatiotemporal bounded noises and transitions induced by them in solutions of the real Ginzburg-Landau model," Physical Review E: Statistical, Nonlinear, and Soft Matter Physics, vol. 86, no. 2, Article ID 021118, 2012.

[43] S. De Franciscis and A. D’Onofrio, "Spatio-temporal sineWiener bounded noise and its effect on Ginzburg-Landau model," Nonlinear Dynamics, vol. 74, no. 3, pp. 607-613, 2013.

[44] S. De Franciscis and A. D’Onofrio, "Cellular polarization: Interaction between extrinsic bounded noises and the wavepinning mechanism," Physical Review E: Statistical, Nonlinear, and Soft Matter Physics, vol. 88, no. 3, Article ID 032709, 2013.

[45] J. García-Ojalvo and J. M. Sancho, Noise in Spatially Extended Systems, Springer Science \& Business Media, 2012.

[46] C. R. Doering, Nonlinear parabolic stochastic differential equations with additive colored noise on $R \times R^{+}:$A regulated stochastic quantization. Communications in Mathematical Physics, vol. 109, 1987, 537-561.

[47] C. Robertson, C. Mazzetta, and A. dOnofrio, Regional variation and spatial correlation, P. Boyle and M. Smans, Eds., vol. Chapter 5 of Atlas of Cancer Mortality in the European Union and the European Economic Area, 2008, 91-113. 


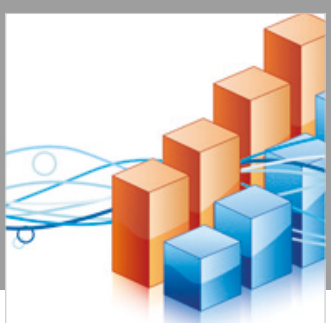

Advances in

Operations Research

\section{-n-m}
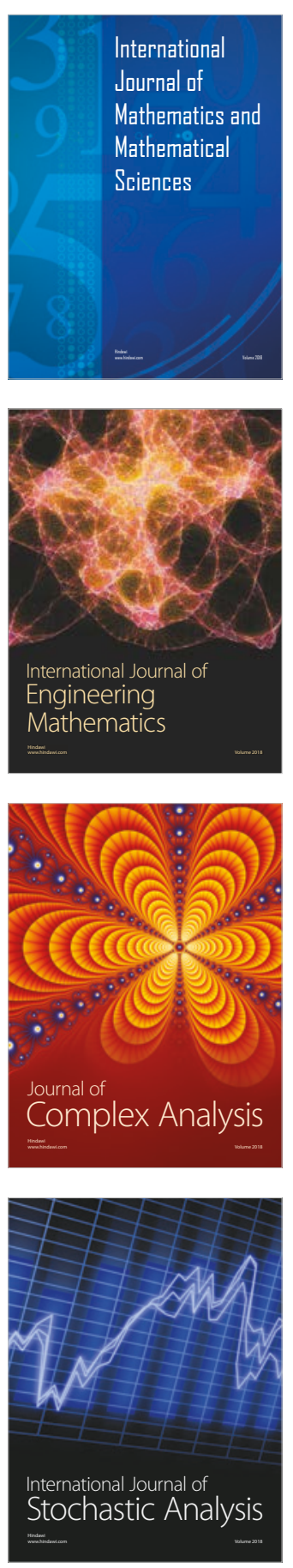
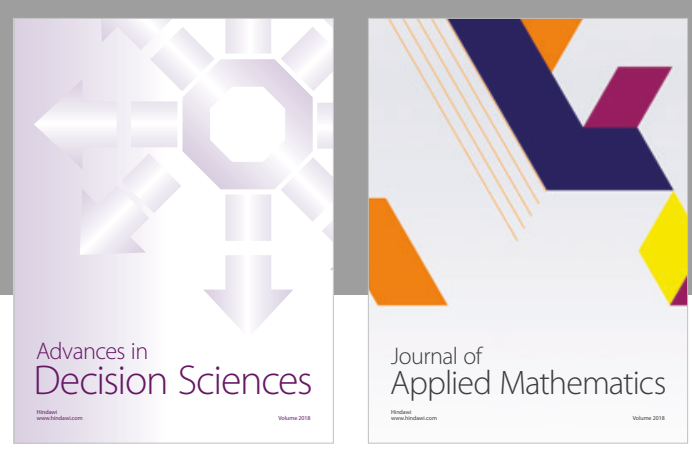

Journal of

Applied Mathematics
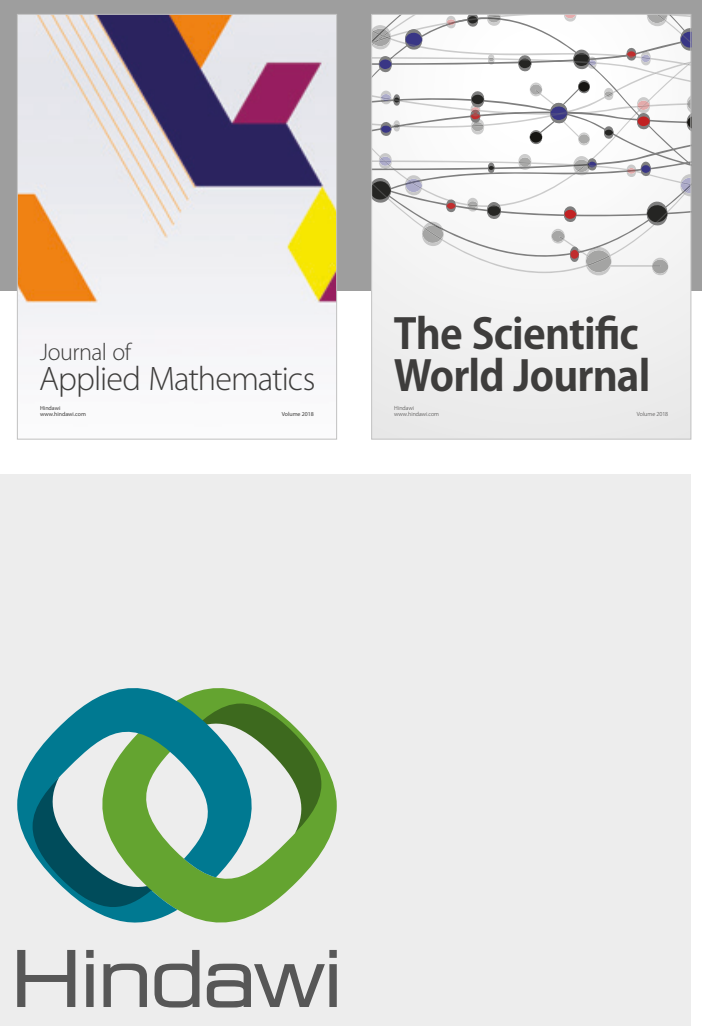

Submit your manuscripts at

www.hindawi.com

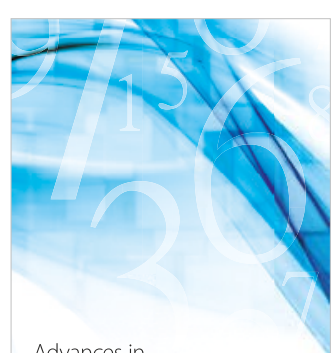

Advances in
Numerical Analysis
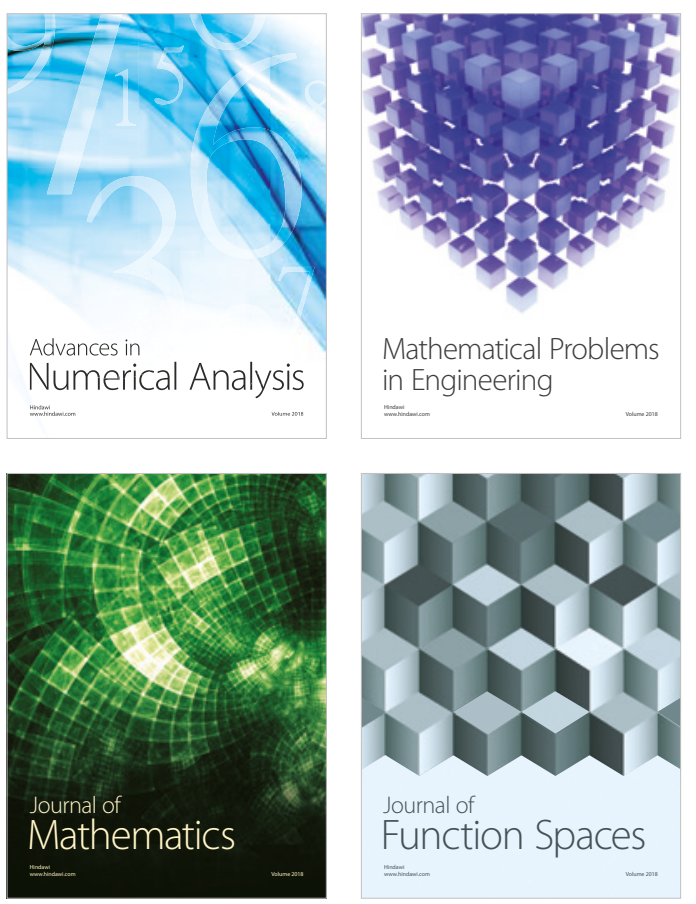

Mathematical Problems in Engineering

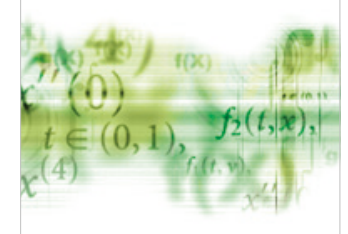

International Journal of

Differential Equations



Journal of

Function Spaces
The Scientific

World Journal

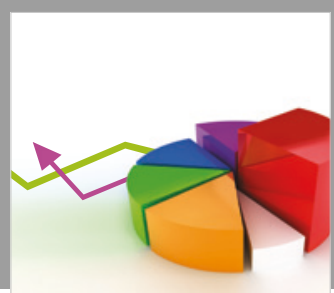

Journal of

Probability and Statistics
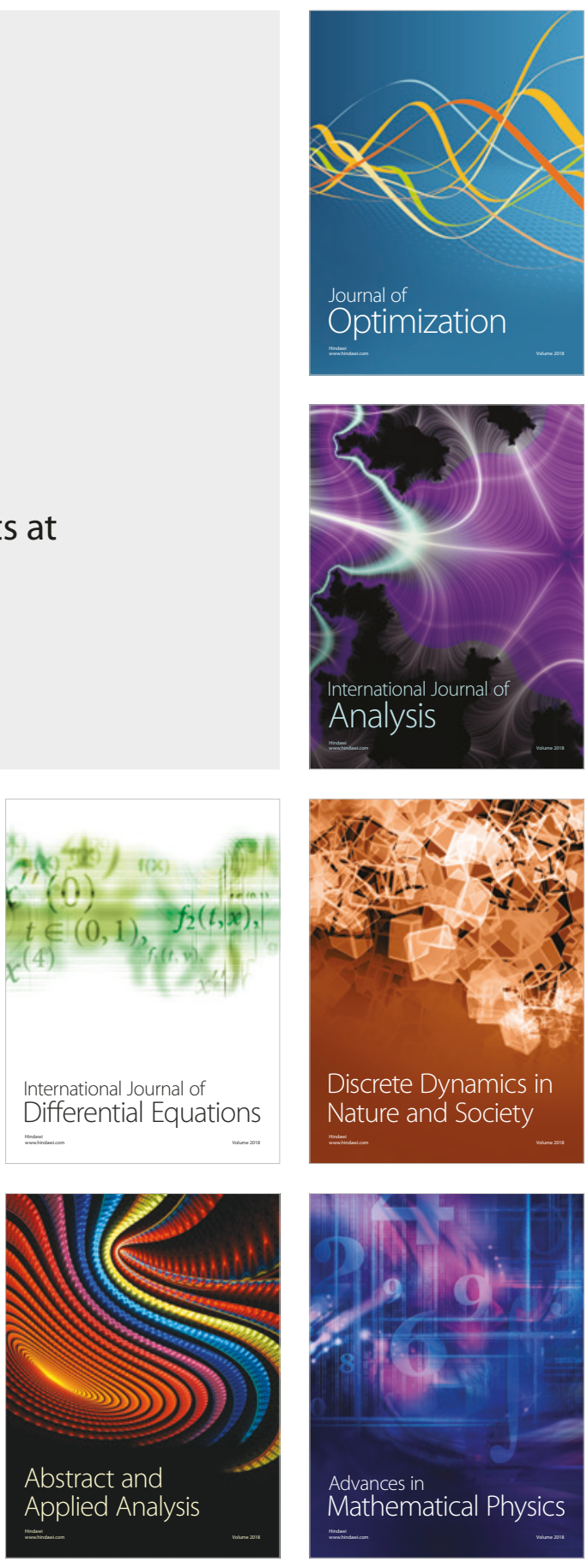